\title{
Susceptibility of Simulium damnosum complex larvae to temephos in the Tukuyu onchocerciasis focus, southwest Tanzania
}

\author{
A.K. KALINGA ${ }^{1}$, C.N. MWEYA ${ }^{1}$, T. BARRO ${ }^{2}$ and B.T.A MAEGGA ${ }^{1 *}$ \\ ${ }^{1}$ National Institute for Medical Research, Tukuyu Research Station, P.O. Box 538, Tukuyu, Tanzania \\ ${ }^{2}$ African Programme for Onchocerciasis Control, B.P. 549, Ouagadougou, Bukina Faso
}

\begin{abstract}
Tukuyu onchocerciasis focus was earmarked for vector control using insecticide against larval stages. Susceptibility tests of mature larvae of Simulium damnosum s.l. vectors to temephos insecticide were carried out before and after two years of insecticide treatment of rivers within Tukuyu onchocerciasis focus, south-western Tanzania. The tests were done in 1999/2000 and 2004 using WHO standard methods. Mature larvae were exposed to 9 concentrations of temephos active ingredient, from the weakest $0.00975 \mathrm{mg} /$ litre to the strongest of $2.5 \mathrm{mg} / \mathrm{l}$. Each test concentration and control was run in duplicates of 25 larvae each, set for three hours in a cool temperature. After incubation, test solution was discarded and larval condition checked. Numbers of larvae in each category were recorded and used to determine mortality rate for each concentration as well as for the $\mathrm{LC}_{50}$ and $\mathrm{LC}_{95}$. A total of 1,666 larvae were tested, 942 during the pre- and 724 post-treatment. Results showed that both pre and post-treatment samples were susceptible, attaining $100 \%$ mortality at the diagnostic dose of $1.25 \mathrm{mg} / 1$, and $\mathrm{LC}_{50}$ between $0.129-0.34 \mathrm{mg} / 1 \mathrm{pre}$ - and $0.144-0.211 \mathrm{mg} / 1(95 \% \mathrm{CI}, P<0.05)$ post- treatment. These values fall within the standard diagnostic dose of $\leq 0.4 \mathrm{mg} / \mathrm{l}$ for susceptible $S$. damnosum s.l populations. It was concluded that the endemic $S$. damnosum population was susceptible to temephos before and after two years of intermittent field application. Temephos was thus recommended for continued use in onchocerciasis vector control in the Tukuyu focus, to complement Community Directed Treatment with Ivermectin, but close monitoring of vector susceptibility should be done.
\end{abstract}

Key words: susceptibility, Simulium damnosum, control, temephos, Tanzania

\section{Introduction}

Onchocerciasis is a disease caused by a filarial nematode Onchocerca volvulus. Adult worms dwell in subcutaneous nodule, from where fertilized females produce millions of microfilariae ( $\mathrm{mf}$ ) which live and freely migrate in the intercellular spaces of the skin tissues, where they cause a wide spectrum of signs and symptoms of skin disease (onchodermatitis). The skin diseases is usually characterised by an acute itching and pruritis which may progress to chronic skin changes leading to loss of elasticity, skin thickening (elephant or lizard skin) and premature ageing. The chronic scratching may lead to pigment changes as in "leopard skin" or depigmentation and dispigmentation. Other manifestations associated with onchocerciasis are hanging groins, hernia and elephantiasis. When the microfilariea invade the eye tissues, they cause damage to different parts of the eye including the cornea, conjunctiva, anterior and posterior eye segments, which may lead to a range of eye defects from loss of visual acuity to irreversible blindness (Buck, 1974). Due to these life long chronic manifestations, the disease is a serious socioeconomic impediment, as it occurs in fertile river valleys most suited for agricultural exploitation.

Onchocerciasis is a tropical disease, endemic in 28 countries in Africa, 6 in Latin America and in the Yemen. An estimated 18 million people are infected, over $99 \%$ of these live in Africa, where the disease is responsible for the loss of 1 million DALYs annually (WHO, 2002). The disease endemic zone stretches across the middle belt of Africa, from Senegal in the west to Ethiopia in the East, on its northern limits and in the south, from Angola to southern Malawi. Tanzania lies within these limits, where the disease epidemiology shows a focal distribution due to the specialized vector ecology.

In 1993, it was estimated that about 650,000 people were infected with $O$. volvulus (WHO, 1995) in different foci of Tanzania, the estimate which has since been updated to over a million since the launching of the national onchocerciasis control programme, which was preceded by country wide rapid epidemiological mapping activities (Ministry of Health Unpublished Document, 1997). Of the historically well known foci, the disease prevalence was reported as high in the Bwakira area of southern Uluguru Mountains (63.6\%), Mahenge (58.6\%), and Ruvuma (31.9\%); and low in Amani (22.4\%) and Tukuyu (22.8\%) (Mwaiko et al., 1990). Previously, a mean onchocerciasis infection rates of $28.7 \%$ (range= $2.9 \%-62.8 .4 \%$ ) had been reported by Pederesen \& Kolstrup (1986).

Onchocerciasis is transmitted by a female black fly of the Simulium species. The intensity of transmission and level of disease endemicity depends 
on the various vector characteristics, from a suitable ecology and population dynamics of the vector as well as vectorial capacity and human socio-economic activities. The vector black flies are active during daytime and outdoors, so they make contact with their hosts in these circumstances.

Until 1986, the disease control campaigns mainly focused on vector control, due to lack of safe drugs for human use. The current interventions include community directed treatment with ivermectin (CDTI) in the 19 endemic countries including Tanzania. In addition, in three of these, focal vector control / elimination projects were recommended. One such a project was earmarked for the Tukuyu focus, to compliment mass ivermectin treatment, and eliminate the parasite reservoir within a shorter duration than it would otherwise take.

Vector control using temephos was the mainstay of the Onchocerciasis Control Programme in West Africa from 1974 to 2002 (Boatin et al., 1997; Borsboom et al, 2003). The insecticide was considered the most environmentally friendly compound whose end products were biodegradable and left no lasting residues in the aquatic environment. The "Simulium formulation" was $20 \%$ emulsifiable concentrate $(20 \%$ EC), which was sprayed from the air by planes over flying target river stretches, to achieve large coverage in a short time, on weekly basis. The aerial spraying was planned to kill vector larval stages before emerging into adults, thus preventing parasite transmission from one person to another for as long as vector control was effective (Davies, 1994; Walsh"et al., 1981).

The onchocerciasis vector in the Tukuyu focus was first identified as the Kiwira form of the $S$. damnosum complex (Maegga, 1992; Maegga \& Cupp, 1993, 1994), which was later confirmed as Simulium thyolense (Mustapha et al., 2005). The most predominant cytospecies was previously identified as a vector in the Thyolo highlands onchocerciasis focus of Southern Malawi (Vajime et al., 2000). No vector control campaign against onchocerciasis had ever been done in Tanzania before the Tukuyu focus vector elimination trials of 2003 and 2005. However, a possibility for the existence of vector innate tolerance and /or exposure to agricultural insecticides or other ecological determinants could not be ruled out, thus measuring levels of susceptibility of $S$. damnosum s.l. larvae to temephos was an essential prerequisite to making the final decision whether to use the particular insecticide or not, and once adopted, for how long it may continue to be used. The study was therefore carried out to determine the susceptibility of untreated populations of $S$. damnosum s.l. larvae to temephos insecticide prior to vector control campaign, and to monitor the susceptibility levels during the course of the campaign for evidence of insecticide tolerance or resistance evolution in the meantime.

\section{Materials and Methods}

\section{Study area}

Tukuyu Onchocerciasis focus covers Rungwe, Kyela and Ileje districts in south-western Tanzania. The focus is located between $9^{\circ} 05^{\prime} \mathrm{S}$ to $9^{\circ} 45^{\prime} \mathrm{S}$ and $33^{\circ} 20^{\circ} \mathrm{E}$ to $34^{0} 202 \mathrm{E}$, in an area of slightly over $3,000 \mathrm{~km}^{2}$. In 2004 , about 98,641 people in the Tukuyu focus were estimated to be infected and approximately 300,000 were at risk of contracting the infection (Ministry of Health, 2004 unpubl.).

Larval sampling sites where susceptibility test specimen was collected from, were selected from river stretches known to have dense S. damnosum s.l. larval populations during the dry season. These included Rivers Kiwira (at Lema), Mbaka (at Kambasegela) and Lufilyo (at Tapio Bridge). The same points were also used for adult fly catching to monitor transmission of onchocerciasis in the area, as first described by Pedersen \& Maegga (1985). Therefore it was highly probable that the $S$. damnosum s.l. sampled for susceptibility testing belongs to the local vector species, $S$. thyolense.

\section{Study design}

The tests were based on established method of WHO (1981), originally field tried by Mouchet et al. (1977). Five in-situ susceptibility tests were conducted of which three tests were for untreated population as baseline data in year 1999/2000. The other three tests were conducted in 2004 as monitoring for the population under treatment. Larvae were collected from breeding sites while still attached to their supports in icebox and transported to the sub-bases. Larvae were picked off the substrates, and lifted with soft forceps and placed in the test enamel bowls containing about $50 \mathrm{ml}$ test solution. Only $6^{\text {th }}$ and $7^{\text {th }}$ instars (mature larvae) were chosen for the test. At least two replicas were set up for each concentration. S. damnosum s.l. larval instars were morphologically identified by standard keys as described by Freeman \& De Meillon (1953) and Crosskey (1969). Sight identification of mature larvae of sixth and seventh instars was done using a hand lens to check for cuticular setae extending forward to the proleg, and pairs of prominent dorsal - lateral abdominal tubercles or bumps. Confirmation of larval identity after the 
test was done under a low power dissection microscope. Specific characteristics checked for the $S$. damnosum complex were the presence and distribution of the cuticular setation and larval development instar criteria of the presence and size of thoracic black spots.

\section{Susceptibility tests}

Temephos was mixed with test water in $500 \mathrm{ml}$ bottles at $19-22^{\circ} \mathrm{C}$. The solutions of temephos were prepared according to protocol of testing the susceptibility of larvae of $S$. damnosum complex (WHO, 1981, 1983) in ascending series of concentrations of 0.00975 , $0.0195,0.039,0.078,0.156,0.3125,0.625,1.25$, and $2.5 \mathrm{mg} / 1$. The solutions were thoroughly mixed by swirling. The water in which larvae were immersed in the test bowls was carefully tipped out without dislodging the larvae and insecticide solution carefully added. Fifteen minutes intervals were left between different concentrations to allow for harvesting time and scoring of each set after the 3 hours of incubation. The control bowls had a solution of a measured amount of absolute ethanol used in diluting temephos mixed with water. Each test series had two control bowls for comparison. All bowls were appropriately labelled using felt-tipped marker and placed in isothermic cabinet for temperature mitigation.

At the end of the 3 hour incubation period, larvae were separated into three groups of live, moribund and dead. Live larvae were characterized by contracting quickly into "U" shape with the head touching the posterior end when the edge of the bowl was rapped or when larvae touched with forceps. Moribund larvae were identified by their sluggish movement when touched where as dead larvae were recognized by being immobile and not sensitive when touched by forceps. At every concentration, removal of larvae followed a trend live, moribund and finally dead. Each category at each concentration was preserved in $80 \%$ alcohol in separate tubes. Final sorting and counting of live, moribund and dead larvae were done in the laboratory with the aid of dissection microscope. Only the mature larvae of $6^{\text {th }}$ and $7^{\text {th }}$ instars were included in test counts, while those that were too young or those classified as pharate pupae were rejected, the separation was done based on morphological criteria for larvae inclusion in these test. At times larvae of species other than S. damnosum s.l. , especially $S$. hargreavesi, were also included as often it was difficult to separate them by sight alone. These were also discarded once confirmed by microscopy.

\section{Data analysis}

Percentage mortality of larvae was calculated from moribund and dead larvae for each set of tests. In tests replicas where control mortality exceeded $5 \%$, but was less than $20 \%$, the test mortalities were corrected by Abbott's formula shown below:

$\%$ corrected mortality $=\%$ test mortality $-\%$ control mortality $\times 100$ $100 \%$ - \% control mortality.

When the control mortality exceeded $20 \%$ the whole test was discarded and the exercise repeated after correction of any presumed errors. PoloPlus software package (version 1.0, LeOra Software, 2002-2006) was used to determine $\mathrm{LC}_{50}$ from the dosage mortality curve. $\mathrm{LC}_{50}$ up to $0.4 \mathrm{mg} / 1$ is accepted value for susceptible population whereas $\mathrm{LC}_{50}$ from $0.4 \mathrm{mg} / \mathrm{l}$ to $1.0 \mathrm{mg} / \mathrm{l}$ indicates intermediate susceptibility, and $\mathrm{LC}_{50}$ above $1.0 \mathrm{mg} / 1$ is a sign of resistance.

\section{Results}

A total of 1,666 larvae of $S$. damnosum complex, (assumed to belong to $S$. thyolense), of $6^{\text {th }}$ and $7^{\text {th }}$ instars were tested. Out of these, 942 were a sample before treatment and 724 after treatment. Samples before treatment were divided as follows: 405 from Mbaka, 158 from Lufilyo and 379 from Kiwira. For the follow-up samples, the breakdown was: 422 were from Mbaka, 302 larvae were from Lufilyo. During the latter study, there was no enough $S$. damnosum s.l. of the right size on the Kiwira river site.

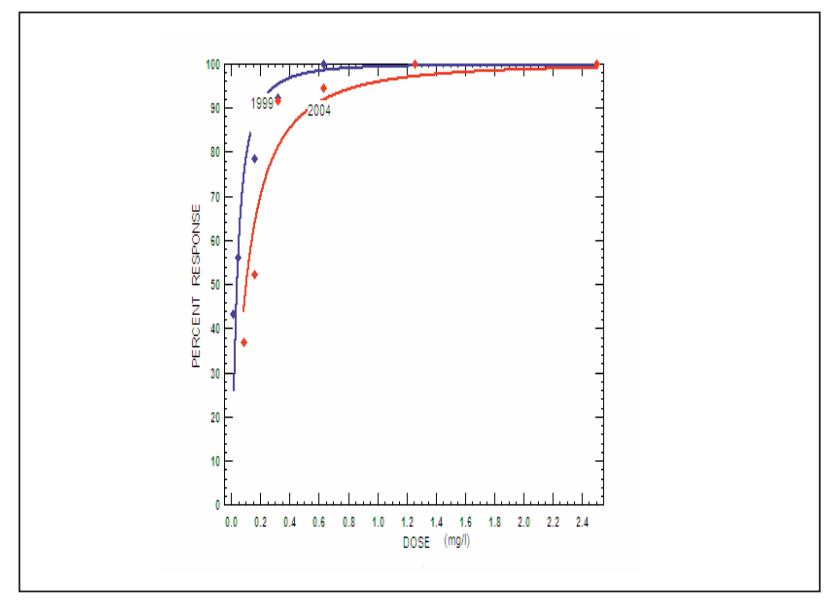

Figure 1: susceptibility to temephos: Percent mortality of mature larvae of $S$. damnosum s.l. at different concentrations in the Mbaka River, Tukuyu focus 


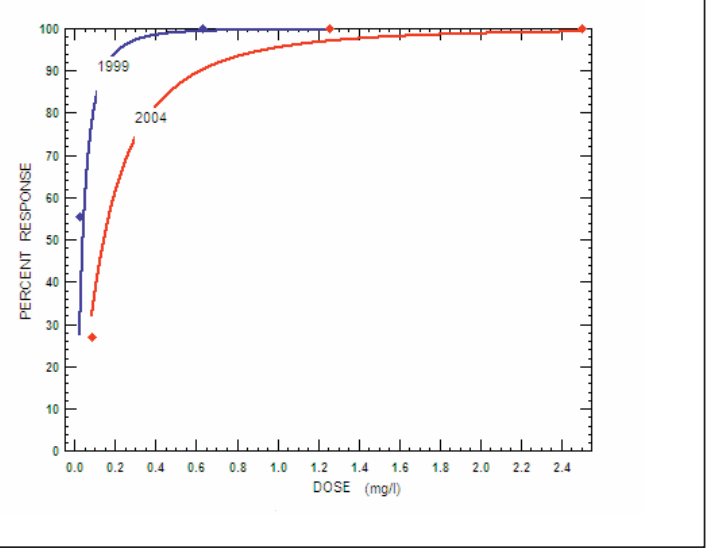

Figure 2: Percent mortality of mature larvae of $S$. damnosum s.l. at different concentrations in the Lufilyo River

In the pre-treatment samples, mortality rate of $100 \%$ was attained with $1.25 \mathrm{mg} / 1$, which is the standard diagnostic dose for $S$. damnosum s.l. vector species. The $\mathrm{LC}_{50}$ was observed to range from $0.129-0.34 \mathrm{mg} /$ $1(95 \% \mathrm{CI}, P<0.05)$, this too was within the standard dose of $\leq 0.4 \mathrm{mg} / 1$ for a susceptible population. After two years of intermittent treatment of the area with temephos, larval mortality rate of $100 \%$ was attained with the diagnostic dose limit of $1.25 \mathrm{mg} / 1$, as for the pre-treatment population. The post-treatment $\mathrm{LC}_{50}$ was $0.144-0.211 \mathrm{mg} / 1(95 \% \mathrm{CI}, P<0.05)$, which was within the standard dose of $\leq 0.4 \mathrm{mg} / 1$ for a susceptible population, as in the pre-treatment case. The mean LC $_{50}$ was highest in Kiwira $(0.211 \mathrm{mg} / 1)$, followed by Lufilyo (0.165mg/1) and Mbaka (0.079mg/1) (Figures $1,2$ and 3$)$.

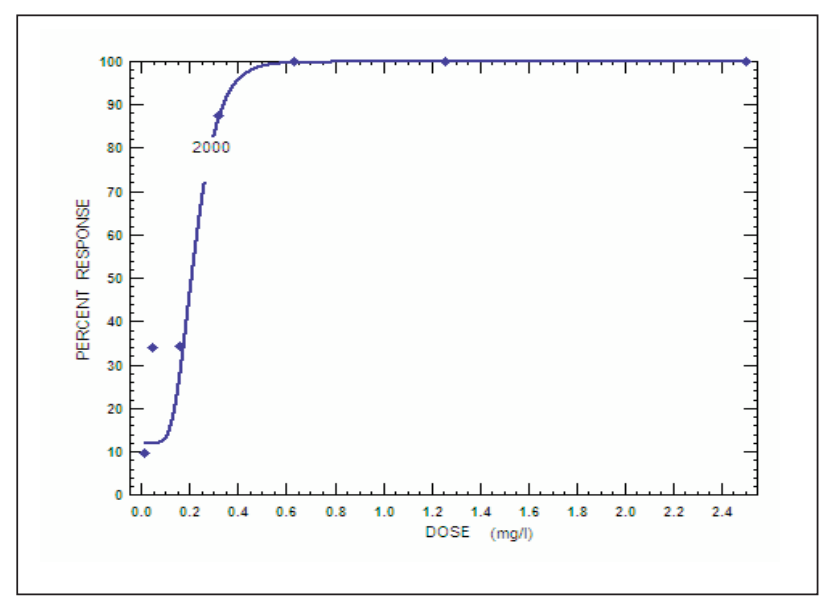

Figure 3: Percent mortality of mature larvae of $S$. damnosum s.l. at different concentrations in the Kiwira River

\section{Discussion}

Tukuyu area has a history of agricultural use of organophosphate pesticides, although concrete documentation was hard to find. Thus there was genuine concern of some of these pesticides or their residues trickling into the drainage system, such as springs, streams and eventually into the river systems which form breeding habitats of the Simulium species, some of which are important vectors of human onchocerciasis. The persistent presence of such insecticides in the normal ecological habitat could potentially induce insecticide tolerance to both target and non-target aquatic fauna. For this reason, it was an essential pre-requisite to determine levels of susceptibility of $S$. damnosum s.l. vectors to this new insecticide, before making final decision to use it as the main control tool, otherwise, the decision could prove counter productive if the insecticide was tolerated by the vector species. However, from the results of the pre- and post-treatment susceptibility tests, it was observed that $S$. damnosum s.l larval populations in the local rivers were very susceptible to the insecticide, and thus, could be used for its control. The indicators of a susceptible population used in these observations were: the lethal concentration range of $0.129-0.34 \mathrm{mg} / 1$ for $50 \%$ mortality of the vector population before treatment was within the standard dosage of $(\leq 0.4 \mathrm{mg} / \mathrm{l})$, as well as that of the post-treatment population. From the experiences of the Onchocerciasis Control Programme (OCP) areas, mature larvae of $S$. damnosum s.l. were regarded to be resistant to temephos when they survive concentrations higher than $1.0 \mathrm{mg} / 1$ (Davies, 1994).

In the current study, $100 \%$ mortality was attained at a relatively low concentration of $0.65 \mathrm{mg} / \mathrm{l}$ ), a clear demonstration of the population's susceptibility to temephos $20 \%$ EC. From the first set of results of the susceptibility tests conducted on Mbaka, Lufilyo and Kiwira rivers within the focus, the decision was made to organise insecticide treatment campaigns against the local vector, using temephos $20 \% \mathrm{EC}$. Then a relatively large scale field vector control feasibility study was conducted for six weeks, from late December 2001 to late January 2002 (data shown elsewhere). After achieving a successful outcome of the large scale feasibility study, the second exercise, which was the first actual vector control campaign was done for 17 weeks in the main Tukuyu focus rivers (20 weeks in Lumbira on the focal fringes), in 2003. In both occasions, vector population was decimated, and the adult biting catches were reduced to undetectable low levels on the three main rivers of 
the Tukuyu focus. However, as insecticide treatment was stopped before completed vector elimination was attained, vector breeding slowly begun, and adult human biting females reappeared after several months (data not shown). The post- treatment susceptibility test was therefore done on the vector population that was found following the first actual control campaign. Again, this population was found to be susceptible to temephos insecticide, as shown by the results. Subsequent to these findings, a second large scale insecticide treatment campaign was executed, for 11 weeks of insecticide spraying into rivers to clear Simulium vector larvae, while simultaneously carrying out rigorous monitoring of the vector breeding and biting activities.

From the experience in West African OCP programme, the first evidence of $S$. damnosum s.l. resistance development to temephos was reported approximately five years after continuous intensive application in the upstream parts (WHO, 1995), while the resistant population was found in downstream stretches. That implies that a diluted concentration of the product was reaching downstream populations for a relatively long time before resistance was detectable. In the current study, the insecticide was not continuously used throughout the two to three years' of treatment, but intermittently, for some weeks and then stopped. The re-colonization of breeding sites could have occurred through residual population left during treatment operations or new unexposed populations migrating from nearby areas. The population's duration of exposure to insecticide pressure was probably not long enough for clear resistance development. On the other hand, the intermittent insecticide treatment approach was a useful strategy in delaying resistance development. For instance, in the vector control campaigns in parts of the OCP, this strategy was used in order to ease the intensity of insecticide selection pressure for resistance development, and even after 8 years of intermittent treatment on the Volta River at Sencchi rapids, the local $S$. damnosum s.l. was found to be still susceptible to temephos (Adiamah, 1986). Thus intermittent insecticide treatment in onchocerciasis vector control could also offer a viable alternative strategy where insecticide resistance development is a real threat. In spite of the satisfactory susceptibility levels observed, it is important to note that different genetic populations of the same $S$. damnosum complex show different paces of resistance development with the same insecticide exposure duration (Davies, 1994). This is based on the inherent genetic variability of the different cytotypes, as they are endowed with natural flexibility to withstand or adapt to different ecological niches in their natural habitats. In addition, in many parts of Africa, including Tanzania, environmental pollution from products used in agriculture, livestock and mining industries cannot be ruled out. Since in most cases, no records are available, extreme vigilance is absolutely essential. For instance, in this study, among the three rivers where larvae were sampled, Kiwira river population showed higher mean $\mathrm{LC}_{50}$ of $0.211 \mathrm{mg} / 1$ compared to Mbaka $(0.079 \mathrm{mg} / \mathrm{l})$ and Lufilyo $(0.165 \mathrm{mg} / \mathrm{l})$, for the untreated population. The reason for this phenomenon was not immediately clear. However, the presence of a coal mining factory nearby the sampling area on the Kiwira river bank may have exposed its population to some chemical products emanating from the factory since its opening over 10 years ago. Whatever the reason for these differences, it was apparent that the Kiwira population showed higher tolerance to temephos than Lufilyo or Mbaka populations. Furthermore, it was also observed that, while the posttreatment susceptibility tests showed a normally susceptible population, the dose- mortality curve slope was slightly less steep, tending towards greater survival at concentrations lethal for the pre-treatment population. Further investigations are therefore recommended to follow-up this phenomenon very closely.

\section{Acknowledgements}

We sincerely acknowledge the technical, financial and material support of the African Programme for Onchocerciasis Control, Ouagadougou, Burkina Faso, for facilitating this study. We are grateful to the National Institute for Medical Research (NIMR), Tanzania for the permission to carry out the study and for the provision of human power. The diligent work of field technicians, especially A. Mwaikonyole, A Kibweja, D. Charle, M. Kibona and the driver S. Msamila of NIMR, Tukuyu Station is greatly appreciated. Permission to publish these results was given by the Director General, NIMR, Dar-es-Salaam, for which we are most grateful.

\section{References}

Adiamah, J.H., Raybould, J.N., Kurtak, D., Israel, A., Maegga, B.T.A. \& Opoku, A.K. (1986).The susceptibility of Simulium damnosum s.1 larvae from the Volta River in southern Ghana to temephos after nearly eight years of 
intermittent treatment. Insect Science and its Application 7, 27-30.

Boatin, B., Molyneux D.H., Hougard J.M., Christensen O.W., Alley E.S., Yameogo L., Seketeli A., and Dadzie K.Y. (1997). Patterns of epidemiology and control of onchocerciasisi in West Africa. Journal of Helminthology 71: 91-101

Borsboom, G.J.J.M., Boatin B.A., Nigelkerke N.J.D., Agoua H., Akpoboua K.L.B. , Alley E.W.S., Bissan Y., Renz A., Yameogo L., Remme J.H.F. and Habbema J.D.F. (2003). Impact of ivermectin on onchocerciasis transmission. Assessing the empirical evidence that repeated ivermectin mass treatment may lead to elimination / eradication in West Africa. Filaria Journal (http:// www.filariajournal.com/content/2/1/8) March18, 2003 pp 36+12 figs.

Buck, A.A. (1974). Onchocerciasis: symptomatology, pathology, diagnosis. World Health Organization Geneva 80 pp.

Crosskey, R.W. (1969) A reclassification of the Simuliidae (Diptera) of Africa and its Islands. Bulletin of the British Museum (Natural History) Entomology Supplement 14. London. 195 pp.

Davies, J.B. (1994). Sixty years of onchocerciasis vector control: a chronological summary with comments on eradication, reinvasion and insecticide resistance. Annual Review of Entomology 39, 23-45.

Freeman, P. \& De Meillon, B. (1953) Simuliidae of the Ethiopian Region. British Museum (Natural History): vii +224 pp.

Maegga, B.T. (1992) Identification of Simulium damnosum Vectors of Onchocerca volvulus in the Tukuyu Valley, Southwest Tanzania. PhD Thesis, Cornell University, Ithaca, New York, USA. xiv + 269 pp.

Maegga, B.T.A \& Cupp, E.W. (1993) Chromosomal diagnostic criteria for some members of Simulium damnosum complex in East Africa. Tropical Medicine and Parasitology 44,165171.

Maegga, B.T.A. \& Cupp, E.W. (1994) Cytotaxonomy of the Simulium damnosum complex and description of new cytotypes in the Tukuyu Focus, southwest Tanzania. Tropical Medicine and Parasitology 45, 125-129.

Ministry of Health, Tanzania (1997). Proposed National Plan for Control of Onchocerciasis in Tanzania. Unpublished document for submission to African Programme for
Onchocerciasis Control (APOC) 1 February, 1997. 44pp + 33pg Tables.

Mouchet, J., Quelennec, G., Beri D. Sechan, Y. \& Grebaut, S. (1977) Méthodologie pour tester la sensibilité aux insecticides des larves de Simulium damnosum s.1.: Cahiers ORSTOM serié Entomologie Médicale et Parasitologie 15, 55-66.

Mustapha, M.A., Krueger, A., Tambala, P.A.J. \& Post, R.J. (2005) Incrimination of Simulium thyolense (Diptera: Simuliidae) as the anthropophilic blackfly in the Thyolo focus of human onchocerciasis in Malawi. Annals of Tropical Medicine \& Parasitology 99, 181192.

Mwaiko, G.L., Mtoi, R.S. \& Mkufya, A.R. (1990) Onchocerciasis prevalence in Tanzania. Central African Medical Journal 36, 94-96.

Pedersen, E.M. \& Maegga, B.T.A. (1985) Quantitative studies on the transmission of Onchocerca volvulus by Simulium damnosum s.1. in the Tukuyu Valley, southwest Tanzania. Tropical Medicine and Parasitology 36, 49-254.

Pedersen, E.M. \& Kolstrup, N. (1986) The epidemiology of onchocerciasis in the Tukuyu valley, southwest Tanzania. Tropical Medicine and Parasitology 37, 35-38.

Vajime, C.G., Tambala, P.A.J., Krueger, A. \& Post, R.J. (2000) The cytotaxonomy of Simulium damnosum s.l. (Diptera: Simuliidae) from the Thyolo onchocerciasis focus in Malawi and description of a new member of the complex. Annals of Tropical Medicine \& Parasitology 94, 279-290.

Walsh, J.F., Davies, J.B. \& Cliff, B. (1981) World Health Organization Onchocerciasis Control Programme in the Volta River Basin. In: Marshall- Laird (Ed.) Blackflies: The Future for Biological Methods in Integrated Control. Academic Press London. Pg 85-115.

WHO (1981) Manual: Instructions for determining the Susceptibility or Resistance of Black fly Larvae to Insecticides. (WHO/VBC/81.811):

WHO (1983) Manual: Protocol for Testing the Susceptibility of Larvae of Simulium damnosum s.l. to Insecticides as used in Vector Control Unit of the Onchocerciasis Control Programme (OCP). WHO/VBC/81. 811

WHO (1995) Onchocerciasis and its Control. Report of WHO Expert Committee on Onchocerciasis control. WHO Tech. Rep. Ser. 852 . 